\title{
Evaluating Relational Ranking Queries Involving Both Text Attributes and Numeric Attributes
}

\author{
Liang Zhu' ${ }^{1}$, Zhaoliang Xie ${ }^{1}$, Qin $\mathrm{Ma}^{2}$
}

\begin{abstract}
${ }^{1}$ Key Laboratory of Machine Learning and Computational Intelligence, School of Mathematics and Computer Science, Hebei University, Baoding, Hebei 071002, China; ${ }^{2}$ Department of Foreign Language Teaching and Research, Hebei University, Baoding, Hebei 071002, China

Email: zhu@hbu.edu.cn, xiezhaoliang533@163.com, maqin@hbu.edu.cn
\end{abstract}

Received October 12, 2012

\begin{abstract}
In many database applications, ranking queries may reference both text and numeric attributes, where the ranking functions are based on both semantic distances/similarities for text attributes and numeric distances for numeric attributes. In this paper, we propose a new method for evaluating such type of ranking queries over a relational database. By statistics and training, this method builds a mechanism that combines the semantic and numeric distances, and the mechanism can be used to balance the effects of text attributes and numeric attributes on matching a given query and tuples in database search. The basic idea of the method is to create an index based on WordNet to expand the tuple words semantically for text attributes and on the information of numeric attributes. The candidate results for a query are retrieved by the index and a simple SQL selection statement, and then top- $N$ answers are obtained. The results of extensive experiments indicate that the performance of this new strategy is efficient and effective.
\end{abstract}

Keywords: Relational Database; Ranking Query; Semantic Distance; Numeric Distance; WordNet

\section{Introduction}

A relational ranking query (or top- $N$ query) is to find the $N$ tuples that satisfy the query condition the best but not necessarily completely, and the results are sorted according to a given ranking function. Researches on top- $N$ queries have intensified since late 1990s [1-3], and most of the researches involve numeric attributes and use a numeric distance function (say, $L_{p}$-norm distances, $p=1,2$, and $\infty$ ) to reduce a massive result set of a conventional query to a few of the most relevant answers. However, there are many applications where ranking queries involving both text attributes and numeric attributes are available for processing.

Example 1. Consider a database of used books with schema: Books(id\#, title, price, year,...). Suppose a customer wants to buy a used book with title on "film", price around " $\$ 50$ " and year about " 2000 ", where title is a text attribute with semantics, and price and year are two numeric attributes. Obviously, a book on "algebra" with price $=$ " $\$ 50$ " and year $=$ " 2000 " is not the desired result for the customer though the values for price and year exactly match that of the query respectively. Another book on "movie", however, with price $=$ "\$53" and year = "2001" maybe satisfy the need of the cus- tomer.

Ipum99
\begin{tabular}{|c|c|c|c|}
\hline$i d x$ & $A 29$ & $A 40$ & $A 50$ \\
\hline 87197 & 42 & 2 & 20000 \\
\hline 6505 & 28 & 3 & 25000 \\
\hline $\mathbf{8 0 7 8 9}$ & $\mathbf{5 1}$ & $\mathbf{9 3 0}$ & $\mathbf{3 3 0 0 0}$ \\
\hline 10860 & 9 & 999 & 999999 \\
\hline
\end{tabular}

Occ50
\begin{tabular}{|c|c|}
\hline num & occ50 \\
\hline 2 & $\begin{array}{c}\text { Airplane pilots and } \\
\text { navigators }\end{array}$ \\
\hline 3 & Architects \\
\hline $\mathbf{9 3 0}$ & $\begin{array}{c}\text { Gardeners, except } \\
\text { farm, and } \\
\text { groundskeepers }\end{array}$ \\
\hline 999 & N/A (blank) \\
\hline
\end{tabular}

Figure 1. Parts of IPUMS Census Database.

Example 2. As shown in Figure 1, database IPUMS has two relations Ipum99 and Occ50, which come from [4]. In relation Occ50(num, occ50), its primary key num is the value label of occupation1950. Relation Ipum99 has 61 numeric attributes where A29 is age, A50 means income, and $A 40$ is the foreign key referencing Occ50.num. Furthermore, Ipum99 is added an attribute $i d x$ by us as identifier. A user is looking for the information of " $a$ horticulturist with age about 50" from IPUMS. Since there is no such word "horticulturist" in Occ50.occ50, the answer will be nothing by using the 
traditional SQL selection statement. In fact, there is a tuple ("Gardeners, except farm, and groundskeepers", age $=51, \ldots$ ) (with gray color) in IPUMS may be an answer for the user.

WordNet::Similarity [5] is an open source Perl module for measuring the semantic distance/similarity between two words; however, it is not easy for us to use the source directly to evaluate ranking queries [6].

Researches on semantic search in IR and SW (semantic web) have gained attention since 1990s. Taking thesaurus ontology navigation as a step in query expansion, many query expansion techniques are employed in keyword search, say, query terms are expanded to WordNet synonyms and meronyms, using the Boolean OR operations available in most web search engines [7]. Different from the above query expansion techniques in IR and SW, we discuss semantic match between query words and tuple words in relational databases via tuple expansion.

In recent years, ontologies have been used to build applications in database community [8, 9]; however, it is a challenging job to construct efficient ontologies $[9,6]$. Our method is different from the ontology-based techniques. Firstly, instead of dealing with the challenge of constructing ontology, we create a simple table maintained by RDBMS, and store information of index and ranking function into the table. Secondly, our strategy is more general since it is based on WordNet. Finally, our techniques are more efficient than the above ontology-based methods due to the efficiency of RDBMS.

Another different yet related research topic is keyword search in relational databases [10-12], which supports free-form keyword search in relational databases without necessarily requiring the users to know the database schema. Keyword search may be suitable for any text attribute, but it is exact search without dealing with semantic match. For instance, it cannot return the books on "movie" for the query keyword "film". If query words match exactly tuple words, the results of semantic search will contain those of keyword search, and improve the effectiveness of keyword search by using our method.

There are two challenging problems for evaluating the type of relational ranking queries in this paper. The first is how to design a good semantic distance function that measure the semantic similarity between the query words and the tuple words, [6] presented a solution for this problem. The second is how to combine the semantic and numeric distances. We employ statistics and training to solve the problem, and create an index to process ranking queries in terms of semantic and numeric matching in database search. Moreover, this work is a continuation of the work in [6], which studied the processing of relational ranking queries only with text attributes, without numeric attributes.

\section{Problem Definition and Ranking Function}

Assume that $\boldsymbol{R}_{0}\left(i d x, A, B_{1}, B_{2}, \ldots, B_{m}, \ldots\right)$ is a relation with identifier $i d x$, where $A$ is a text attribute with natural language semantics and $B_{1}, B_{2}, \ldots, B_{m}$ are $m$ numeric attributes. Using the project operation $\pi_{A}\left(\boldsymbol{R}_{\mathbf{0}}\right)$ (duplicate tuples are eliminated), we get a new relation $\boldsymbol{R}($ tid, $A)$ with identifier tid. Furthermore, $\boldsymbol{R}_{\mathbf{0}}$ is added by an attribute FKid that is the foreign key referencing $\boldsymbol{R}$.tid, and then the schema of $\boldsymbol{R}_{\mathbf{0}}$ becomes $\boldsymbol{R}_{\mathbf{0}}\left(i d x, A, B_{1}, B_{2}, \ldots\right.$, $\left.B_{m}, \ldots, F K i d\right)$. Or, let $\boldsymbol{R}(t i d, A)$ and $\boldsymbol{S}\left(i d x, B_{1}, B_{2}, \ldots\right.$, $B_{m}, \ldots, F K i d$ ) be two relations and $\boldsymbol{S}$.FKid be the foreign key referencing $\boldsymbol{R}$.tid, then $\boldsymbol{R}_{\mathbf{0}}=\boldsymbol{R} \bowtie \boldsymbol{S}$ with $\boldsymbol{S}$.FKid = R.tid.

Let $\boldsymbol{t}$ be a tuple in $\boldsymbol{R}_{\mathbf{0}}$, then $\boldsymbol{t}[A]=\left(t w_{1}, t w_{2}, \ldots, t w_{n}\right)$ is the word-string with $n$ words on the text attribute $A$, and $\boldsymbol{t}\left[B_{j}\right]=b_{j}$ is the numeric value on the attribute $B_{j}(1 \leq j \leq$ $m)$. For simplicity, we denote $\boldsymbol{t}=\left(\boldsymbol{t}_{A}, b_{1}, b_{2}, \ldots, b_{m}\right)$ where $\boldsymbol{t}_{A}=\left(t w_{1}, t w_{2}, \ldots, t w_{n}\right)$, and call $t w_{i}$ a tuple word and $b_{j}$ a tuple value $(1 \leq i \leq n, 1 \leq j \leq m)$.

As defined in [6], given a tuple word $w$, its kinship words include the five kinds of words in WordNet: (1) word $w$ itself, (2) morph, (3) synonyms, (4) the immediate hyponyms (subordinates), and (5) the immediate hypernyms (superordinates). The set of all kinship words of $w$ is denoted by $K(w)$. For instance, the kinship word set of "computers" is $K$ (computers) $=$ \{computers, computer, data processor, machine, internet site, calculator, .... .

Consider a ranking query $\boldsymbol{q}=\left(\boldsymbol{q}_{A}, q_{1}, q_{2}, \ldots, q_{m}\right)$, where $\boldsymbol{q}_{A}=\left(q w_{1}, q w_{2}, \ldots, q w_{k}\right)$ is a word-string with $k$ query words, and $q_{j}$ is a numeric value $(1 \leq j \leq m)$. For tuple $\boldsymbol{t}=\left(\boldsymbol{t}_{A}, b_{1}, b_{2}, \ldots, b_{m}\right)$ in $\boldsymbol{R}_{\mathbf{0}}$, denote $p_{j}=\left|q_{j}-b_{j}\right|(1 \leq$ $j \leq m)$, and $d_{A}=d\left(\boldsymbol{q}_{A}, \boldsymbol{t}_{A}\right)$ that is the semantic distance between $\boldsymbol{q}_{A}$ and $\boldsymbol{t}_{A}$ defined by Definition 1 to 3 in [6]. Moreover, $d_{A}$ belongs to the interval $(0,1]$.

We need to find a mechanism combining the semantic and numeric distances $d_{A}$ and $p_{j}$ s, which can be used to evaluate the query $\boldsymbol{q}$ over the relation $\boldsymbol{R}_{\mathbf{0}}$, i.e., to define a ranking function $d(\boldsymbol{q}, \boldsymbol{t})=\psi\left(d_{A}, p_{1}, p_{2}, \ldots, p_{m}\right)$.

Intuitively, the ranking function $d(\boldsymbol{q}, \boldsymbol{t})$ should satisfy the following: First, a smaller $d(\boldsymbol{q}, \boldsymbol{t})$ indicates closer the pair $(\boldsymbol{q}, \boldsymbol{t})$. Second, $d(\boldsymbol{q}, \boldsymbol{t})$ needs to balance the effects of $d_{A}$ and $p_{j}$ in matching $\boldsymbol{q}$ with $\boldsymbol{t}$. Finally, it should be easy to implement.

To obtain $d(\boldsymbol{q}, \boldsymbol{t})$, we use the statistics of the domains of $B_{1}, B_{2}, \ldots, B_{m}$ and training.

Since the semantic distance $d_{A} \in(0,1]$, we normalize it by scientific notation, $d_{A}=\alpha \times 10^{-h}$, where $1.0 \leq \alpha$ $<10.0$, and $h$ is a nonnegative number (i.e., $h \geq 0$, if $h=0$, 
we define $-0=0$ ), say, $d_{A}=0.001041=1.041 \times 10^{-3}$. We will see that $h$ (the absolute value of the exponent) plays an important role in the ranking function $d(\boldsymbol{q}, \boldsymbol{t})$.

In collecting statistics, there is a step of cleaning data and removing outlier, and then we get $\operatorname{Min}\left(B_{j}\right)$ and $\operatorname{Max}\left(B_{j}\right)$ of numeric attribute $B_{j}(1 \leq j \leq m)$. Based on the semantics of attribute $B_{j}$, we obtain its reasonable unit $Z_{j}>0$, say, $Z_{\text {year }}=1$ for attribute year and $Z_{\text {price }}=0.01$ for price respectively in database $\mathbf{B O O K}$.

Let $M_{j}=\operatorname{Max}\left(B_{j}\right)-\operatorname{Min}\left(B_{j}\right)=\beta_{j} \times 10^{c_{j}}$, where $1.0 \leq$ $\beta_{j}<10.0\left(M_{j} \geq 0\right.$, obviously. If $M_{j}=0$, let $M_{j}:=Z_{j}$ then $M_{j}>0$ ). If $M_{j} \geq 1$, we have $c_{j} \geq 0$, else if $0<M_{j}<1$, let $M_{j}:=M_{j} / Z_{j}$, and $p_{j}:=p_{j} / Z_{j}$, then $M_{j} \geq 1$ and $c_{j} \geq 0$.

Let $e_{j}:=c_{j}+1 \geq 1$, for $1 \leq j \leq m$. By using the statistics and training in our experiments, we obtain the ranking function $d(\boldsymbol{q}, \boldsymbol{t})=\psi\left(d_{A}, p_{1}, p_{2}, \ldots, p_{m}\right)$ below.

$$
d(\boldsymbol{q}, \boldsymbol{t})=d_{A}+\Sigma_{j=1}^{m}\left(p_{j} / M_{j}\right)^{h / e_{j}}
$$

For example, in our experiments for relation Books (title, price, year,...) with 56180 tuples. price is in $(0$, 1000] (except 633 tuples with price $>1000$ ), and year belongs to [1958, 2008] (except 651 tuples with year < 1958). Let attribute $A, B_{1}$ and $B_{2}$ be title, price and year respectively, then $M_{1}=1000-0=1000=1.0 \times 10^{3}, M_{2}$ $=2008-1958=50=5.0 \times 10^{1}$, and then $c_{1}=3, c_{2}=1, e_{1}$ $=c_{1}+1=4$ and $e_{2}=c_{2}+1=2$. Thus, $d(\boldsymbol{q}, \boldsymbol{t})=d_{A}+$ $\left(p_{1} / 1000\right)^{h / 4}+\left(p_{2} / 50\right)^{h / 2}$.

For query $\boldsymbol{q}$, we will return dis- $N$ tuples defined in [6] to replace top- $N$ ones. Let $\boldsymbol{T}$ be a set of tuples, a tuple $\boldsymbol{t} \in \boldsymbol{T}$ is called a dis- $N$ tuple of $\boldsymbol{q}$, if $d(\boldsymbol{q}, \boldsymbol{t}) \leq \min _{N}\left\{d\left(\boldsymbol{q}, \boldsymbol{t}_{i}\right)\right.$ $\left.\mid \boldsymbol{t}_{i} \in \boldsymbol{T}\right\}$, which means the $N$ th minimum value in the set $\left\{d\left(\boldsymbol{q}, \boldsymbol{t}_{i}\right) \mid \boldsymbol{t}_{i} \in \boldsymbol{T}\right\}$.

\section{Creation of sn-Index}

We extend w-index in [6] to sn-index (stands for semantic and numeric index) in this section.

\subsection{Information Stored in sn-Index}

A relation is employed to store the information in sn-index, which is called IndexTable with schema IndexTable(id\#, Word, Size, dbNSize, DBValue, BValue, bSize), say, (2490, nights, 2, 2, '47898,0,3,1;2413,0,...', '47898,8185,10.80,1997;...', 3) is a tuple in IndexTable for database BOOK.

The attribute Word indicates the kinship word of tuple words, and the relevant information will be stored in the attribute DBValue. The value of DBValue is a string with form "tid,d,n,f;tid,d,n,f;...;" where "tid,d,n, $f ;$;" is a node, $d=0,1, \ldots, 5$, " $d=i$ " is the subscript $i$ of semantic distance $d_{i}$ defined by Definition 1 in [6] , $n$ is the number of tuple words in the tuple with tid, and $f$ is the frequency in the set of kinship words of the tuple. The attribute Size is the number of tids associated with $k w$, and the duplicate tids are counted repeatedly. The attribute $d b N S i z e$ means the number of nodes in $D B V a-$ lue.

Attribute BValue is a string as "tid,idx, $b_{1}, b_{2}, \ldots, b_{m}$; tid,idx, $b_{1}, b_{2}, \ldots, b_{m} ; \ldots$, ;, where " tid,idx $, b_{1}, b_{2}, \ldots, b_{m}$;" is a node, tid is the identifier of a tuple in the relation $\boldsymbol{R}(t i d$, $A)$, and $i d x$ is that in $\boldsymbol{R}_{\mathbf{0}}(i d x, \ldots)$. The attribute bSize means the number of nodes in BValue.

\subsection{Procedure of Creating sn-Index}

Reconstructing w-index in [6], we obtain sn-index as shown in Figure 2. Sn-index consists of four parts: (1) a hash table with a hash function $h()$, its each bucket contains a pointer $p W n$ Pointer which points to a node in wn-list; (2) one wn-list, its node has structure \{iRow, $k w$, size, dbNSize, bSize, $p D B L i s t, p B L i s t\}$; (3) $s$ db-lists, the structure of the node is $\{t i d, d, n, f\}$; (4) $s$ b-lists, node structure $\left\{t i d, i d x, b_{1}, b_{2}, \ldots, b_{m}\right\}$. The main difference between sn-index and w-index in [6] is that b-lists (i.e., $B_{i j}$ 's in Figure 2) are added. The algorithm of creating sn-index is shown as follows:

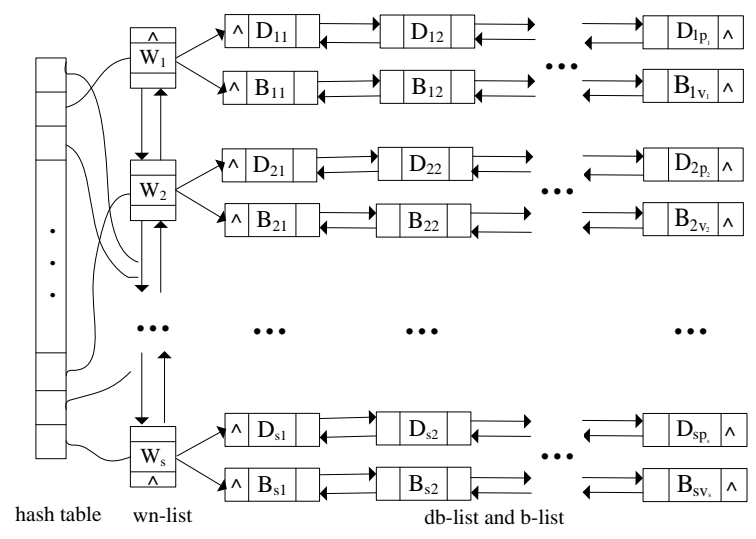

Figure 2. Structure of sn-index.

Step 1. For the special character string $\boldsymbol{t}_{A}=\left(t w_{1}, \ldots\right.$, $\left.t w_{n}\right)$, calculate its hash value $h=h\left(t_{A}\right)$; check the pointer $p W n$ Pointer in the bucket of the hash table, and wn-list,

(1) If $p W n$ Pointer is NULL, or $\left(t w_{1}, \ldots, t w_{n}\right)$ is not in wn-list,

(a) Create a new db-list, and a dbNode as tid $=$ $\boldsymbol{t}_{\mathrm{A}}$.tid, $d:=-1$ (means the special case), $n:=1$ and $f:=1$. Insert the dbNode into the db-list.

(b) Using tid above, search (idx, $\left.b_{1}, b_{2}, \ldots, b_{m}\right)$ from $\boldsymbol{R}_{\mathbf{0}}$ (or from $\boldsymbol{S}$ ) by the following the SQL selection statement

Select idx, $\mathrm{B}_{1}, \mathrm{~B}_{2}, \ldots, \mathrm{B}_{\mathrm{m}}$ from $\mathbf{R}_{\mathbf{0}}$ where FKid $=$ tid 
Let $z$ be the size of the result set $\left\{\left(i d x, b_{1}\right.\right.$, $\left.\left.b_{2}, \ldots, b_{m}\right)\right\}$, Create a new b-list, and $z$ bNode(s), insert the bNode(s) into the b-list.

(c) Create a wnNode for wn-list, $k w:=\left(t w_{1}, \ldots\right.$, $\left.t w_{n}\right)$, and $d b N S i z e:=1$ and bSize:= $z$. Insert the wnNode into wn-list according to the alphabet order of $k w$.

(d) Get the pointers pDBList, pBList and pWnPointer.

(2) Else if the string $\boldsymbol{t}$ is found out in wn-list, then increase $d b N S i z e$ by 1 in the wnNode with $\boldsymbol{t}$, create a dbNode as above, and let it be the first node of the db-list. Additionally, by the SQL selection statement as (b) above, get a result set $\left\{\left(i d x, b_{1}\right.\right.$, $\left.\left.b_{2}, \ldots, b_{m}\right)\right\}$ with size $z \geq 1$. Create $z$ bNode(s), insert the node(s) into the b-list, and increase $b$ Size by $z$.

Step 2. For each tuple word $t w_{i} \in \boldsymbol{t}$, and each kinship word $k w$ of $t w_{i}$, get $h=h(k w)$, check $p W n P o i n t e r$ in the bucket of the hash table, and wn-list,

(1) If $p W n P o i n t e r$ is NULL, or $k w$ is not in wn-list, do the same jobs as the above (1) in Step1 except for replacing $\boldsymbol{t}_{\mathrm{A}}$ by $k w$ and defining $d=k, k$ $\in\{0,1, \ldots, 5\}$ such that $d_{k}$ is $d\left(k w, t w_{i}\right)$ in Definition 1 in [6].

(2) Else if $k w$ has been in wn-list, then get its db-list and b-list. There are two cases.

Case 1 , in the db-list, there is a dbNode with dbNode.tid $=t$ tid, replacing $d$ by the smaller if the two distances are different, and increasing $f$ by 1 , that is $\mathrm{OK}$.

Case 2, if no dbNode in the db-list satisfies dbNode.tid $=$ t.tid, increase $d b N$ Size by 1 in the wnNode with $k w$, create and insert a new dbNode into the db-list according to the increasing order of distance $d$. In addition, by the SQL selection statement as (b) above, get a result set $\left\{\left(i d x, b_{1}, b_{2}, \ldots, b_{m}\right)\right\}$ with size $z \geq 1$. Create $z$ bNode(s), insert the node(s) into the b-list, and bSize:= $z$.

Step 3. Storing sn-index.

To evaluate query $\boldsymbol{q}$, we use two storing strategies. Strategy-1, the entire sn-index is in main memory. Strategy-2 will store db-lists and b-lists in fixed disk and only load the hash table and wn-list into main memory.

\section{Evaluation of Ranking Query}

For query $\boldsymbol{q}=\left(\boldsymbol{q}_{A}, q_{1}, q_{2}, \ldots, q_{m}\right)$, firstly, matching the query words with kinship words of $\boldsymbol{R}($ tid, A) via sn-index, we obtain the set $\boldsymbol{T}$ (= $\{$ tid $\}$ ) of identifiers of candidate tuples, and compute the semantic distances between $\boldsymbol{q}$ and its candidate tuples, and then get the set $\boldsymbol{L}$ of information of numeric attributes by each identifier tid in $\boldsymbol{T}$; secondly, compute $d(\boldsymbol{q}, \boldsymbol{t})=\psi\left(d_{A}, p_{1}, p_{2}, \ldots, p_{m}\right)$ between $\boldsymbol{q}$ and each of its candidate tuples, and then obtain $\{i d x\}$, which is the sorted set of identifiers of dis- $N$ results according to $d(\boldsymbol{q}, \boldsymbol{t})$; finally, we retrieve the dis- $N$ tupels from underlying database and display the ranked answers.

To get the set $\boldsymbol{T}$ is an important step for query processing. The intermediate results are stored in a temporary list, denoted by T-List. Its node has the structure \{tid, $\left.d[K], d_{A}\right\}$ where $K$ is the maximum number of query words for $\boldsymbol{q}_{A}=\left(q w_{1}, \ldots, q w_{k}\right)$. If $k>K$, let $\boldsymbol{q}_{A}=$ $\left(q w_{1}, \ldots, q w_{K}\right)(K=30$ in our implementations). The intermediate results for obtaining set $\boldsymbol{L}$ are saved in a temporary list L-List, and the structure of its node is $\left\{d_{A}\right.$, $\left.p_{1}, p_{2}, \ldots, p_{m}, d, t i d, i d x\right\}$

We discuss Strategy-2 of storage first (i.e., we only load the hash table and wn-list in main memory).

(1) Normalization of $\boldsymbol{q}_{A}$. We remove some symbols, character strings or stop words, and replace some of them by normal strings for $\boldsymbol{q}_{A}$. Denoted again by $\boldsymbol{q}_{A}=$ $\left(q w_{1}, \ldots, q w_{k}\right)$.

(2) For each $q w_{i} \in \boldsymbol{q}_{A}(i=1, \ldots, k)$, calculate hash value $h=h\left(q w_{i}\right)$, and check $q w_{i}$ in wn-list. If $q w_{i}$ is found out, we get the wnNode in wn-list, and then use the SQL selection statement

select $*$ from IndexTable where id\# = wnNode.iRow to obtain dbNode(s). For each dbNode, we save the values into T-List, and compute $d_{A}=d\left(\boldsymbol{q}_{A}, \boldsymbol{t}_{A}\right)$, then obtain $\boldsymbol{T}$.

(3) If above $\boldsymbol{T} \neq \varnothing$, for each tid $\in \boldsymbol{T}$, we get its corresponding bNode(s) by using select statements from Indextable, store them into L-List, compute $d:=\psi\left(d_{A}\right.$, $\left.p_{1}, \ldots, p_{m}\right)$, and then obtain the set $\boldsymbol{L}(=\{i d x\})$ of candidate dis- $N$ tuple identifiers.

(4) Given a positive integer $N$, we get the set of dis- $N$ tuple identifiers $\boldsymbol{L}_{N} \subseteq \boldsymbol{L}$; then, we obtain the dis- $N$ tuples of the query $\boldsymbol{q}$ by using the SQL selection statement as the following format:

Select R0.*, R.A from R0, R where $(\mathrm{R} 0$. FKid = R.tid $)$ and (R.idx in $\boldsymbol{L}_{N}$ ) and ...

(5) Display the dis- $N$ tuples sorted by $d(\boldsymbol{q}, \boldsymbol{t})$.

Next, we discuss Strategy-1 of storage, i.e., the entire w-index is saved into main memory. The query processing is similar to the above situations, except for steps (2) and (3), we can get dbNode(s) and bNode(s) from sn-index directly, without using select statements. Thus, the response time of Strategy-1 will be smaller than that of Strategy-2.

Example 2 (cont.). For the dis-5 query "a horticulturist with age $=50$ and income $=30000$ " against IPUMS, the answers with form (idx, $\boldsymbol{t}_{\boldsymbol{A}}$, age, income, $\left.d(\boldsymbol{q}, \boldsymbol{t})\right)$ are

$\boldsymbol{t}_{1}$ : (80789, $\left.\boldsymbol{t}_{A}, 51,33000,0.609496\right)$,

$\boldsymbol{t}_{2}:\left(07296, \boldsymbol{t}_{A}, 48,25000,0.729997\right)$,

$\boldsymbol{t}_{3}:\left(77380, \boldsymbol{t}_{\mathrm{A}}, 37,30600,0.747815\right)$, 
$\boldsymbol{t}_{4}:\left(43851, \boldsymbol{t}_{\mathrm{A}}, 51,21894,0.763280\right)$, and

$\boldsymbol{t}_{5}:\left(73792, \boldsymbol{t}_{A}, 47,25000,0.766698\right)$,

where $\boldsymbol{t}_{\boldsymbol{A}}=\boldsymbol{t}[$ occ50] = "Gardeners, except farm, and groundskeepers".

\section{Experimental results}

Our experiments are carried out using Microsoft's SQL Server 2000 and VC++6.0 on a PC with Windows XP, Intel(R) Core(TM) i5-2400/3.10GHz 3.09GHz CPU, and 2.98GB memory. In addition, WordNet 2.1 and its API functions, ODBC, and ODBC API functions are used.

Datasets: We use two real datasets. The first one is IPUMS with two relations that is a fragment of US Census Bureau data [4]. The relation Occ50(num, occ50) contains 286 tuples with 2 attributes. The relation Ipum99(idx, FKnum, age, income, ...) has 61 attributes and 88443 tuples, where age is A29, income is A50, and FKnum is $A 40$ that is the foreign key referencing Occ50.num. $\boldsymbol{R}_{0}=$ Ipum99 $\bowtie$ Occ50 with FKnum = num. Part of IPUMS is shown in Example 2 in Section 1.

The second dataset BOOK comes from the Library at Beijing University of Technology, which is the record set of English books in the Library, and produces two relations. One is Books(id\#, isbn, title, author, publisher, price, year, FKid) having 56180 tuples. The other relation Titles(tid, title) has 48107 tuples (duplicate titles are removed). In addition, Books and Titles act as $\boldsymbol{R}_{\mathbf{0}}$ and $\boldsymbol{R}$ respectively. Books.id\# corresponds to $\boldsymbol{R}_{\mathbf{0}} . i d x$, and Books.FKid is the foreign key referencing Titles.tid.

Attributes tid, $A, B_{1}$, and $B_{2}$ described in Section 2 correspond num, occ50, age and income for IPUMS, and tid, title, price and year for BOOK, respectively.

Space cost of sn-index: Strategy-1 and Strategy-2 are used for IPUMS and BOOK respectively. The main memory space costs are: index-space-IPUMS is about 3.6MB, and index-space-BOOK about 3MB.

Workloads: We build a program to create a workload that is a set of 100 queries for each database. First, we choose 100 tuples from $\boldsymbol{R}$ randomly, and then for each tuple $\boldsymbol{t}$, select $1 \sim 10$ kinship word(s) from $K(t)$ randomly, where the numbers of simple queries with $1 \sim 3$ words and complex queries with $4 \sim 10$ words are both 50 . We classify them into 10 groups $G_{i}(i=1,2, \ldots, 10)$, and the query in $G_{i}$ has $i$ search word(s). The size of $G_{i}$ is random.

For the legends in the following figures, the suffixes “ 1 ”, “2”, ..., and " 100 ” indicate the dis- 1 , dis-2, .., and dis-100 queries, respectively.

\subsection{Elapsed Time}

Figure 3 illustrates the average elapsed time for executing all queries in each $G_{i}$ for IPUMS by fourteen curves $i t \mathrm{~N}$ 's and $d t \mathrm{~N}$ 's, which stand for index-time's and
DB-time's respectively. The seven curves it1 to it100 are (almost) the same, and are related to queries, but not to $N(N=1,2, \ldots, 100)$, which are from 11 to 230 milliseconds. The other seven curves $d t 1$ to $d t 100$ are the costs of retrieving tuples from DB by using SQL selection statements for the natural join of Ipum99 and Occ50, which are smaller than 600 milliseconds.

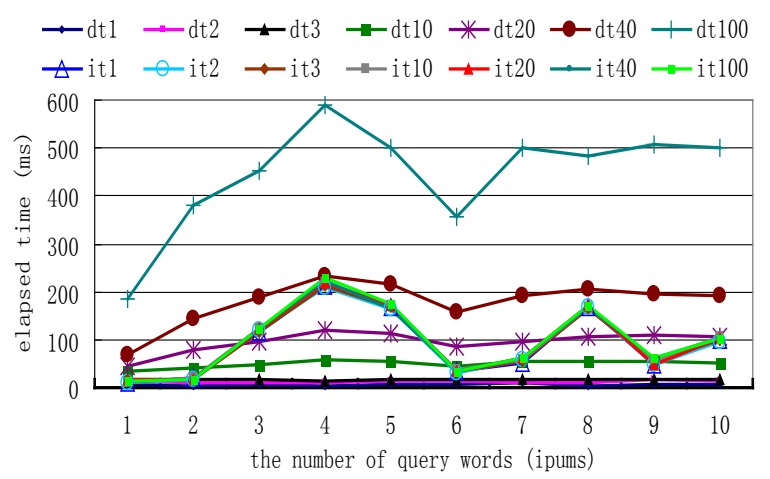

Figure 3. The elapsed times for IPUMS

Figure 4 shows the elapsed time with fourteen curves for BOOK. The seven curves it1 to it100 are (almost) the same, and are related to queries, but not to $N(N=1$, $2, \ldots, 100$ ), which are between $34 \mathrm{~ms}$ and 346ms. Curves $d t 1$ to $d t 100$ show the average elapsed times for accessing database to retrieve tuples. The result sets are different for various dis- $N$ queries. The larger $N$ means the longer the elapsed time. The DB-times are less than 20 ms for $1 \leq N \leq 3$. If $10 \leq N \leq 100$, the DB-times are between 50 and $300 \mathrm{~ms}$.

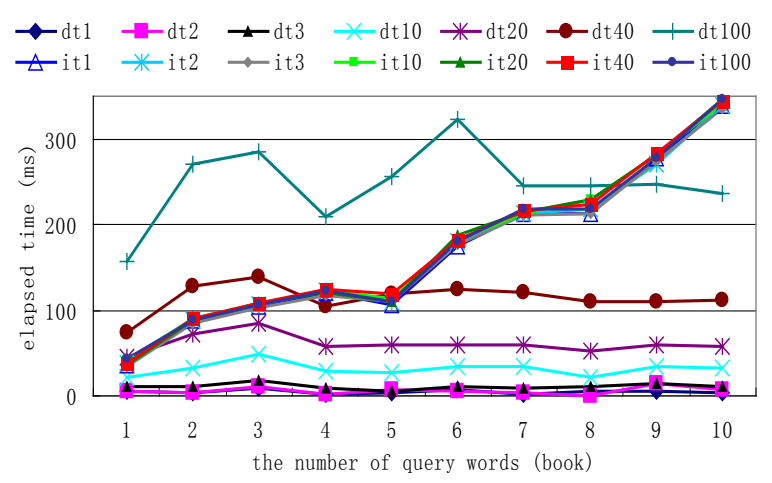

Figure 4. The elapsed times for BOOK

\subsection{Precision}

It is difficult to confirm whether one of tuples retrieved matches a query semantically by a computer, and it is too big a job to recognize semantic match manually for a large dataset [6]. Therefore, the traditional recall for evaluating IR systems is not suitable for measuring semantic match when the dataset is large, and then we 
report only precision. Figures 5 and 6 illustrate the average precision for IPUMS and BOOK respectively.

We can see that a smaller $N$ has a larger precision; therefore, a smaller $N$ indicates more matching tuples appear in its dis- $N$ results. The precisions for IPUMS are $1,0.93,0.90,0.73,0.62,0.52$, and 0.44 for $N=1,2$, $3,10,20,40$, and 100 , respectively. The precisions for BOOK are $0.93,0.86,0.78,0.63,0.55,0.45$, and 0.34 for $N=1,2,3,10,20,40$, and 100 , respectively.

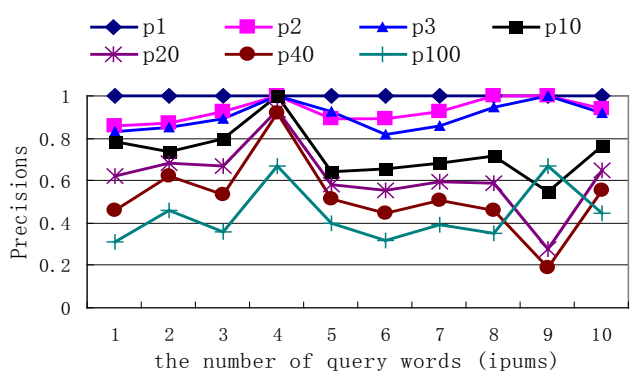

Figure 5. Precisions for IPUMS

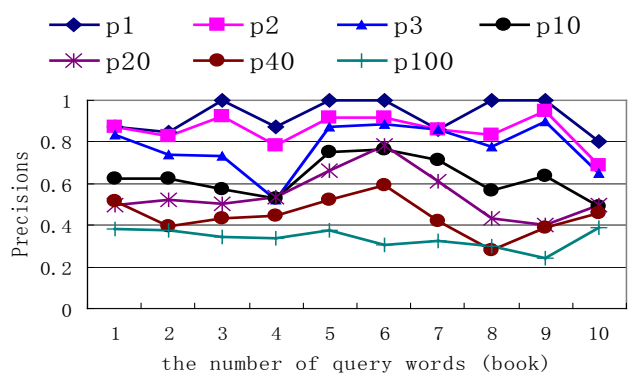

Figure 6. Precisions for BOOK

\section{Conclusions}

We proposed a new method to evaluate relational ranking queries that reference both text attributes and numeric attributes. The method builds a ranking function combining the semantic and numeric distances, and creates an index based on WordNet to expand the tuple words semantically for a text attribute and on the information of numeric attributes. Thus, the results for a query are retrieved by the index and a simple SQL selection statement for the natural join of relations, and ranked according to the ranking function. We conducted extensive experiments to measure the performance of this new technique using two real datasets. The results of experiments demonstrated that our strategy is efficient and effective.

\section{Acknowledgements}

This work is supported in part by NSFC (61170039) and the NSF of Hebei Province (F2012201006). The authors would also like to express their gratitude to Professor Weiyi Meng for providing many helpful suggestions.

\section{REFERENCES}

[1] M. Carey, and D. Kossmann, "On saying 'enough already!' in SQL," SIGMOD 1997 Proceedings ACM international conference on management of data, Vol. 26 No. 2, 1997, pp. 219-230.

[2] I. F Ilyas, G. Beskales, and M. A. Soliman, “A survey of top-k query processing techniques in relational database systems,” ACM Computing Surveys, Vol. 40, No. 4, 2008, Article 11.

[3] L. Zhu, W. Meng, C. Liu, W. Yang, and D. Liu, "Processing top-N relational queries by learning," Journal of Intelligent Information Systems. Vol.34, No.1, 2010, pp.21-55, doi:10.1007/s10844-009-0078-7.

[4] IPUMS Census Database, “ipums.la.99.gz”, 1999, http://kdd.ics.uci.edu/databases/ipums/ipums.html

[5] T. Pedersen, “WordNet::Similarity,” 2008, http://www.d.umn.edu/ tpederse/similarity.html

[6] L. Zhu, Q. Ma, C. Liu, G. Mao and W. Yang. "Semantic-distance based evaluation of ranking queries over relational databases," Journal of Intelligent Information Systems, Vol. 35, No. 3, 2010, pp. 415-445.

[7] D. Buscaldi, P. Rosso, and A. E. Sanchis, "A wordnet-based query expansion method for geographical information retrieval," Working Notes for the CLEF Workshop, Vienna Austria, 2005.

[8] S. Das, E. Chong, G. I. Eadon, and J. Srinivasan, "Supporting ontology-based semantic matching in RDBMS," Proceedings of the Thirtieth International Conference on Very Large Data Bases (VLDB'04), Toronto, Canada, 2004, pp. 1054-1065.

[9] J. Zhang, Z. Peng, S. Wang and H. Niehang, "Si-SEEKER: Ontology-based semantic search over databases," Knowledge Science, Engineering and Management, Guilin, China, Vol. 4092, 2006, pp. 599-611.

[10] V. Hristidis, L. Gravano, and Y. Papakonstantinou, "Efficient IR-style keyword search over relational databases," In Proceedings of 29th International Conference on Very Large Data Bases (VLDB'03), Berlin, Germany, 2003, pp. 850-861.

[11] F. Liu, C. Yu, W. Meng and A. Chowdhury, "Effective Keyword Search in Relational Database," $26^{\text {th }} A C M$ SIGMOD/PODS international Conference on Management of Data/Principle of Database Systems, Chicago, Illinois, USA, 2006, pp. 563-574.

[12] L. Zhu, Y. Zhu, and Q. Ma, "Chinese Keyword Search over Relational Databases," World Conference on Science and Engineering (WCSE), Wuhan, China, Vol. 1, 2010, pp. $217-220$. 